\title{
Identification and Characterization of the Core Rice Seed Microbiome
}

\author{
Alexander W. Eyre, ${ }^{1,2}$ Mengying Wang, ${ }^{1,2}$ Yeonyee Oh, ${ }^{1,2}$ and Ralph A. Dean ${ }^{1,2, \dagger}$ \\ ${ }^{1}$ Center for Integrated Fungal Research, North Carolina State University, Raleigh, NC \\ ${ }^{2}$ Department of Entomology and Plant Pathology, North Carolina State University, Raleigh, NC \\ Accepted for publication 17 April 2019.
}

\section{ABSTRACT}

The use of microbes in agriculture for enhancing crop production is an emerging alternative to chemical fertilizers and pesticides; however, their effectiveness is often limited by factors such as host genotype and variability in geographic location. To address this issue, the microbiomes of six different rice (Oryza sativa) seeds, sourced from two locations in Arkansas, U.S.A. of two different genotypes and two harvest years, were characterized. The bacterial and fungal communities were identified in each of four seed compartments (grain, outer grain, husk, and outer husk) using high throughput Illumina MiSeq sequencing. More unique amplicon sequence variants were identified in the outer seed husk and least in the grain compartment for both the fungal and bacterial microbiomes, however this only resulted in a decrease in diversity for the fungal communities. Principal component analysis indicated that each tissue compartment harbored relatively distinct bacterial and fungal communities for the three innermost compartments. A bacterial and fungal core microbiome shared among the six seed types for each compartment was identified. Key bacterial genera in the core across all compartments were Sphingomonas, Methylobacterium, and taxa in the family Enterobacteriaceae, members of which have been reported to support rice growth. Compared with the bacterial core, more fungal taxa were identified, possibly resulting from the more abundant reads after filtering, and key genera identified were Alternaria, Hannaella, and members of the order Pleosporales. These core members represent valuable candidates for manipulating the rice microbiome, decreasing the use of chemicals while increasing plant performance.

Keywords: endophytes, metagenomics, microbiome, microorganism
Seed germination represents the most dynamic stage of a plant's life cycle and a period in which its initial microbiome is established. This collection of microbes is acquired through both vertical (from the parent plant) and horizontal (from the environment) inheritance mechanisms (Gundel et al. 2011; Shade et al. 2017; Vandenkoornhuyse et al. 2015). Upon planting, the seed imbibes water and releases exudates, a collection of carbon- and nitrogenrich compounds. These are competed for by the microbial inhabitants of the seed, its surface, and the surrounding soil to establish the spermosphere, or zone of elevated microbial activity around the seed (Nelson 2004). Studies have demonstrated this community's ability to promote even germination rates through

${ }^{\dagger}$ Corresponding author: R. A. Dean; radean2@ncsu.edu

Funding: Financial support was provided by NCSU Plant Soil Microbial Community Consortium and the NC College of Agriculture and Life Sciences.

*The $\boldsymbol{e}$-Xtra logo stands for "electronic extra" and indicates that three supplementary tables are published online.

The author(s) declare no conflict of interest.

(C) 2019 The American Phytopathological Society phytohormone secretion (Goggin et al. 2015) and protect the emerging seedling from soilborne pathogens when it is in this vulnerable stage (Bacilio-Jiménez et al. 2001; Beckstead et al. 2007). Additionally, these microbes serve as an initial inoculum for the emerging seedling. The extent this early microbial community contributes to seedling establishment and the mature plant microbiome remains unclear (Compant et al. 2010; Hardoim et al. 2012; Huang et al. 2016).

Rice (Oryza sativa) is a staple food for over half of the world's population and has been established as a model plant for research (Kawahara et al. 2013). Several studies have examined the microbiome of rice through both culturing and sequencing methods (Breidenbach et al. 2016; Edwards et al. 2015), including examination of members within its seed (Cottyn et al. 2009; Okunishi et al. 2005). Bacterial genera commonly identified within rice seed include Curtobacterium, Enterobacter, Methylobacterium, Microbacterium, Sphingomonas, and others, many of which are known to promote plant growth through the supply of nutrients or phytohormones (Midha et al. 2016). In addition to the large bacterial communities, rice seeds are associated with fungal communities; however, in depth studies of the latter have received little attention to date. It has been observed that in natural soil grown conditions, the microbiomes of roots decrease in microbial 
abundance and diversity from the root surface to the inner tissues (Edwards et al. 2015).

A number of studies have shown that a large amount of variability exists in the microbiomes between plant species, subspecies, and even between individual plants in a monoculture cropping system (Fitzpatrick et al. 2018; Hamid et al. 2017). Known sources of this variability come from environmental fluctuation, soil composition, soil microbes, and plant genotype. Changes to one or more of these factors may modify the communities in association with the plant or the properties of the microbes' environment within the plant itself, which can lead to alterations in the community composition (Buyer et al. 1999; Hacquard 2016). This raises the question of whether plants do or do not maintain core microbiomes that are independent of environment and other factors. An ideal plant core microbiome is defined as the microbial community shared between several plant genotypes grown in various geographical areas that contain different soil compositions and environmental conditions. The members of the core represent a conserved subset of the plant's microbiome that presumably carry functions of high value for the plant or surrounding microbial community (Engelbrektson et al. 2012; Huse et al. 2012; Vandenkoornhuyse et al. 2015). These would be ideal candidates for additional research, as they should have the most intimate relationships with the plant and its microbiome.

The focus of this research was to deduce a microbiome for rice (Oryza sativa) using six seed types sourced from two different rice genotypes from two separate years and locations in Arkansas, U.S.A., and provide evidence for a core microbiome. Rice cultivation in this area does not rely on a flooded paddy as is often practiced in Asian countries, rather is planted in fields supplemented heavily with water (Baldwin et al. 2011). For each of the six seed types, seeds were separated into four distinct tissue compartments (grain, outer grain, husk, and outer husk) and the microbes extracted from each; however, only single replicates of each were able to be acquired limiting statistical analyses. Microbial profiles were constructed by amplifying the $16 \mathrm{~S}$ rRNA gene (V3-V4 region) in bacteria and the internal transcribed spacer (ITS1) region of fungi, then additional analyses performed to study the effects of each factor and to develop a putative core microbiome.

\section{MATERIALS AND METHODS}

Rice seed and experimental design. Rice (Oryza sativa) seed used for these experiments was obtained from Y. Jia at the USDA Dale Bumpers National Rice Research Center. Six seed types of the japonica cultivar were provided from research fields at the Dale Bumpers (DB) Center and University of Arkansas (UA). Provided seed was harvested from two genotypes (Katy and M202) and from 2 years (2013 and 2014); however, seed from 2013 was not available from UA (Table 1). The samples were sent through standard mail enclosed in envelopes containing $50 \mathrm{~g}$ of seed, and then stored dry at $4^{\circ} \mathrm{C}$ while unused.

Tissue sample collection and DNA extraction. In order to prepare samples for DNA extraction, 80 whole intact rice seeds were selected for each seed type and placed into sterile $50-\mathrm{ml}$ Falcon tubes with $15 \mathrm{ml}$ of PBS buffer (per 1 liter: $8 \mathrm{~g}$ of NaCl, $0.2 \mathrm{~g}$ of $\mathrm{KCl}, 1.42 \mathrm{~g}$ of $\mathrm{Na}_{2} \mathrm{HPO}_{4}, 0.24 \mathrm{~g}$ of $\mathrm{KH}_{2} \mathrm{PO}_{4}$, adjusted to $\mathrm{pH} 7.4$ using $\mathrm{HCl}$ ). Samples were prepared in triplicate. The tubes were vortexed for 2 min to remove any loosely adhering microbes and then the liquid extract decanted and collected. Three 1-min sonications were subsequently performed each with fresh $15 \mathrm{ml}$ of PBS buffer using a sonication probe (Microson Ultrasonic Cell Disruptor model XL2000, Misonix Incorporated, New York) to remove tightly adhering microbes. Then all buffer extracts were pooled with the vortexed buffer extracts to form the outer husk compartment samples (Fig. 1). The husks were removed from the grains with sterile tweezers and separated into sterile Falcon tubes. Both the separated husks and grains were vortexed once for 2 min and sonicated once for $1 \mathrm{~min}$ with fresh $15 \mathrm{ml}$ of PBS, and then the decanted buffer extracts from the husk and grain were pooled to form the outer grain compartment. Buffer extracts were centrifuged at $12,000 \mathrm{rpm}$ for $15 \mathrm{~min}$ and the supernatant was removed from the pellet; respective pellets represented the outer husk and outer grain compartments. The remaining husks and grains were placed in sterile tubes, and all samples were stored at $-20^{\circ} \mathrm{C}$ until DNA extraction (Bulgarelli et al. 2013, 2012; Engelbrektson et al. 2012). DNA was extracted from samples as follows. Husk and grains were placed separately in a sterile mortar and pestle, and liquid nitrogen was added. For outer husk and outer grain samples, $600 \mu \mathrm{l}$ of DNA extraction buffer was added before being transferred to sterile mortar and pestle with liquid nitrogen. The samples were thoroughly ground and the DNA was extracted using Wizard Genomic DNA Purification Kit by Promega (Madison, WI) following the provided instructions (Fadrosh et al. 2014). The six seed samples of each compartment (collected from 80 seeds for each seed type) prepared in triplicate were combined for a single sequencing run.

Preliminary analyses were conducted to evaluate the presence of bacteria and fungi using direct plating. Samples were prepared as described above, except 10 seeds per sample were used. For the outer husk and outer grain compartments, serial dilutions of the supernatant following vortexing and pooling were plated. Washed husks and grains were ground with a sterile mortar and pestle, sterile

TABLE 1

The six rice seed samples and their associated factors

\begin{tabular}{lllc}
\hline Sample & Genotype & Year & Location $^{\text {a }}$ \\
\hline A & Katy & 2013 & DB \\
\hline B & Katy & 2014 & DB \\
\hline C & M202 & 2014 & UA \\
\hline D & M202 & 2013 & DB \\
\hline E & Katy & 2014 & UA \\
\hline F & M202 & 2014 & DB \\
\hline $\begin{array}{l}\text { a Research fields at the Dale Bumpers (DB) Center and University of } \\
\text { Arkansas (UA). }\end{array}$ & &
\end{tabular}

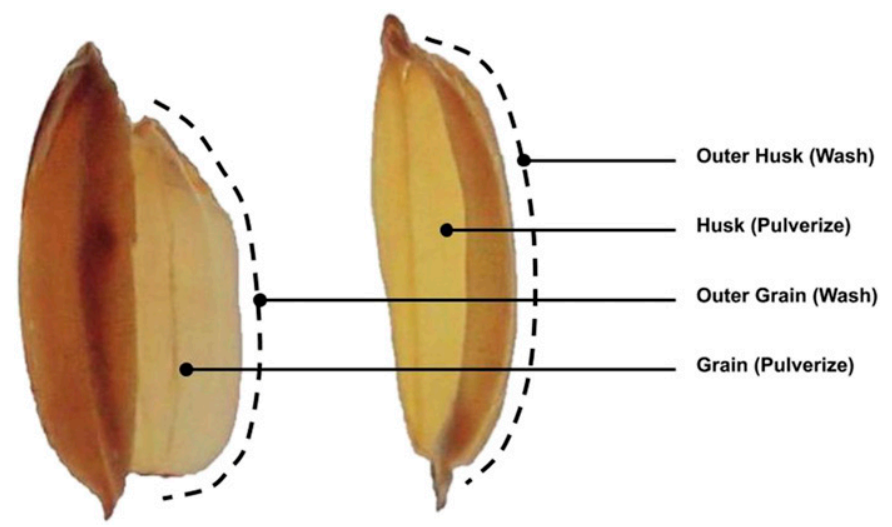

Fig. 1. Rice seed compartments. Illustration of the rice seed and its four tissue compartments for sample collection. Wash indicates the tissue sample was vortexed and sonicated to collect the microbes in solution. Pulverize indicates the tissue sample was frozen and ground. 
water was added, and the solutions were serially diluted. Bacterial presence was confirmed on LB plates incubated at $37^{\circ} \mathrm{C}$ for 1 to 3 days, and fungal presence was confirmed on PDA plates incubated at $25^{\circ} \mathrm{C}$ for 1 to 5 days by visual inspection.

16S V3-V4 and ITS1 PCR amplification and sequencing. In order to identify bacterial members, the V3-V4 region from the bacterial 16S rRNA gene was amplified using the well-established 319F (5'-CCTACGGGNGGCWGCAG-3') and 806R (5'GACTACHVGGGTATCTAATCC-3') primers (Fadrosh et al. 2014; Walters et al. 2015). For examination of fungal members, the ITS1 region from the fungal ITS gene was amplified with the ITS1F (5'-CTTGGTCATTTAGAGGAAGTAA-3') and ITS1R (5'-GCTGCGTTCTTCATCGATGC-3') primers (Usyk et al. 2017). Overhang adapters were attached to the primers for compatibility with the Nextera Index Kit (Illumina, San Diego, CA), and matching adapters were added to both the bacterial and fungal amplifications for a sample.

The DNA quality and concentration were first checked using a Nanodrop Spectrophotometer (model ND-1000, ThermoFisher Scientific, Waltham, MA). Two stages of PCR were conducted. For bacteria, each PCR cycle was performed at $25 \mu \mathrm{l}$ volume containing $25 \mathrm{ng}$ of gDNA, $10 \mu \mathrm{M}$ forward primer, $10 \mu \mathrm{M}$ reverse primer, and $12.5 \mu \mathrm{l}$ of KAPA HiFi HotStart Readymix (Kapa Biosystems, Wilmington, MA). The PCR amplification conditions were as follows: $95^{\circ} \mathrm{C}$ for $3 \mathrm{~min} ; 25$ cycles at $95^{\circ} \mathrm{C}$ for $30 \mathrm{~s}, 55^{\circ} \mathrm{C}$ for $30 \mathrm{~s}$, and $72^{\circ} \mathrm{C}$ for $30 \mathrm{~s} ; 72^{\circ} \mathrm{C}$ for $5 \mathrm{~min}$ and hold at $4^{\circ} \mathrm{C}$. For fungi, each PCR cycle was performed at $25 \mu \mathrm{l}$ volume containing $25 \mathrm{ng}$ of gDNA, $10 \mu \mathrm{M}$ forward primer, $10 \mu \mathrm{M}$ reverse primer, $10 \mu \mathrm{M}$ dNTPs, $0.125 \mu \mathrm{l}$ of Taq DNA Polymerase (New England Biolabs, Ipswich, MA), and $2.5 \mu \mathrm{l}$ of $10 \times$ buffer. The PCR amplification conditions were as follows: $95^{\circ} \mathrm{C}$ for $5 \mathrm{~min} ; 30$ cycles at $95^{\circ} \mathrm{C}$ for $30 \mathrm{~s}, 55^{\circ} \mathrm{C}$ for $30 \mathrm{~s}$, and then $68^{\circ} \mathrm{C}$ for $30 \mathrm{~s} ; 68^{\circ} \mathrm{C}$ for $5 \mathrm{~min}$ and then hold at $4^{\circ} \mathrm{C}$. Following PCR amplification with appropriate barcode adapters, equal proportions of the bacterial and fungal amplifications were pooled for each sample to reduce sequencing costs. Samples were sent to the Genomic Sciences Laboratory at North Carolina State University for Illumina MiSeq 300 bp paired end sequencing (Illumina). All raw amplicon sequence data for this project have been deposited at the NCBI SRA under project 531035 and are available at https://www.ncbi.nlm.nih.gov/sra/PRJNA531035.

Data processing. The raw Illumina reads were initially demultiplexed, and their quality was assessed using FastQC v0.11.5 (https://www.bioinformatics.babraham.ac.uk/projects/fastqc/, Babraham Bioinformatics, Cambridge, UK) in search for passing sequencing and tile quality. The reads for each sample were first divided based on whether they were derived from bacterial or fungal sequences using custom Python scripts parsing for the associated primer sequences. These reads were then uploaded into R v1.1.453 (https://www.r-project.org/), for data processing and analysis. The reads were trimmed, quality filtered, assembled into amplicon sequencing variants (ASVs) using the dada2 v1.8.0 package (Callahan et al. 2016) with default settings, and singletons removed. The trimming protocol called for the removal of primer sequences and a cutoff point within the read that captured the maximum possible length of the corresponding amplicons while avoiding low quality regions (255 bp for the bacteria and $140 \mathrm{bp}$ for the fungi). The 18.70 $\mathrm{M}$ reads provided from the sequencer yielded 853,266 and $4,174,138$ high quality reads for the $16 \mathrm{~S}$ V3-V4 and ITS1 datasets, respectively.

Taxonomic assignment was performed using the dada2 package (Callahan et al. 2016). The 132 release of the SILVA 16S rRNA database (Quast et al. 2013) was used to pick the bacterial taxa utilizing the $99 \%$ database with 7 level taxonomy (https://www.arbsilva.de/). The UNITE ITS version 7.2 database (Abarenkov et al.
2010) was used to pick the fungal ASVs, also utilizing the $99 \%$ database (https://unite.ut.ee/). The $99 \%$ databases were opted for over the others due to the correction of sequencing errors by the dada 2 algorithm that are usually compensated for with clustering to a threshold and selection of a database at that threshold (usually 97\%).

The resulting ASVs from the bacterial assignment were filtered of mitochondrial and chloroplast contaminant sequences to yield 410,687 high-quality bacterial reads. We accounted for differences in sequencing read depth across samples by randomly subsampling (rarefying using default seed) to approximately the minimum number of reads among the samples, corresponding to 465 and 37,000 reads per sample in the $16 \mathrm{~S} \mathrm{V3-V4} \mathrm{rRNA} \mathrm{and} \mathrm{ITS1} \mathrm{datasets,}$ respectively. The overall lower number of reads for the bacteria was largely attributed to the longer bacterial amplicon length and saturation of some grain samples by plant-derived reads (Supplementary Table S1).

Data exploration and statistical analysis. In order to explore the distribution of unique ASVs among the seed samples and compartments, Venn diagrams of the total taxonomic assignments were constructed using the draw.quad.venn function of the VennDiagram v1.6.20 package (Chen and Boutros 2011). To explore the ability to distinguish seed compartments based on the unique ASVs and their abundances, we performed principal component analyses (PCA) using the ordinate command as part of the adaptiveGPCA v0.1.2 package (Fukuyama 2017). Bar charts for the resulting read and ASV abundance data were constructed using the ggplot2 package v3.0.0 (Wickham et al. 2018). Clustering of the taxa containing at least $0.1 \%$ of the total reads was performed using distance matrices derived from the vegdist command of the vegan v2.5-2 package (Oksanen 2015). These were subsequently clustered using the 'complete' method of the hclust command and number of clusters auto assigned. In order to assess the diversity in each seed compartment, the Shannon diversity $\left(\mathrm{H}^{\prime}\right)$ index was estimated using the diversity command as part of the vegan package (Oksanen 2015).

\section{RESULTS}

Microbial communities associated with rice seed. In order to reveal a broad picture of the microbial members within the rice seed, we first analyzed the distribution of unique amplicon sequencing variants (ASVs) by seed compartment. Overall, a total of 296 unique bacterial ASVs and 341 unique fungal ASVs were identified as having more than one read in any one seed compartment. For both bacteria and fungi, several similar trends in the distribution of ASVs were observed (Fig. 2). In both instances, there was a decline in the number of unique ASVs from the exterior (outer husk) to the interior of the seed (grain), with the exception of the outer grain for the fungi. The outer husk contained a majority of the unique ASVs, $65.9 \%$ of the unique ASVs for bacteria and $76.8 \%$ for fungi. Furthermore, for both bacteria and fungi, $>30 \%$ of unique ASVs were found exclusively in the outer husk compartment. In contrast, the grain contained the fewest, $12.5 \%$ for the bacterial and $19.4 \%$ for the fungal ASVs. Of the bacterial members, approximately $62.5 \%$ were found in only a single seed compartment with $37.5 \%$ being shared with at least one other, and only $2.0 \%$ shared between all compartments. Of the fungal ASVs, approximately $51.3 \%$ were found in a single compartment, $48.7 \%$ shared with at least one other compartment, and $12.0 \%$ shared between all compartments. In contrast to the distribution of bacterial ASVs, for fungi the outer grain compartment contained more unique ASVs compared with the husk, which may have biological significance or be a result of the wash methods employed. Additionally, for the bacteria, only 
$16.2 \%$ of the grain compartment was shared with all other compartments, while $66.1 \%$ of the fungal grain compartment was shared with all others.

Analysis of the bacterial V3-V4 reads using the dada2 package (Callahan et al. 2016) and the 132 release of the SILVA 16S rRNA database yielded a total of 157 taxonomic classifications primarily at the genus level. To summarize the distribution of bacterial taxa across the four seed compartments, taxa ranks with at least $1 \%$ of the total reads across all samples were first examined. Nine taxa were identified that contained $95.7 \%$ of the total reads. Figure 3 shows the distribution of reads and the proportion of distinct ASVs in each of the taxonomic groups identified (Fig. 3). Based on read abundance, the class Alphaproteobacteria was found to be most abundant in the three outermost seed compartments ranging from 86.7 to $92.6 \%$, while the class Gammaproteobacteria was most abundant in the innermost seed compartment (77.9\%) and was nearly absent in both the outer grain and outer husk compartments $(\sim 0.2 \%)$. A reduction of diversity from the outer husk (58 ASVs) to the interior of the grain (25 ASVs), particularly a near loss of members of Curtobacterium, was also apparent. Additionally, regardless of variable read abundance in the three outermost tissues, members of the class Alphaproteobacteria maintained relatively similar ASV abundance and retained much of their presence in the grain despite their low read abundance.
For further insight into the bacterial members, those taxa that were present with greater than $0.1 \%$ of the total reads were examined, of which there were 22 taxa in total (Supplementary Table S2). To identify microbial distribution patterns across seed compartments, the extracted data were normalized, subjected to kmeans clustering, and graphically visualized (Fig. 4A). ASVs assigned to cluster A were found predominately in the grain and contained $79.0 \%$ of the grain reads represented by the table. Members of this cluster were primarily in the family Enterobacteriaceae. ASVs in clusters B and E contained the single taxa Asaia and Eukaryota, which were primarily within the outer husk and outer grain compartments, respectively. ASVs in clusters C, D, and $\mathrm{F}$ all contained at least four bacterial taxa and exhibited an increasing abundance from the interior to the exterior of the seed with variable abundance patterns. Bacteria in cluster $\mathrm{C}$ were primarily in low abundance among the compartments $(<1 \%)$ and contained mainly members of the class Alphaproteobacteria. Bacteria in cluster F contained the highly abundant members of the phylum Actinobacteria and two most abundant members of the class Alphaproteobacteria whose abundance patterns, in addition to those in cluster D, were consistent with their patterns in Figure 3.

Analysis of the fungal ITS1 reads employing the UNITE ITS version 7.2 database yielded a total of 319 taxonomic classifications of fungi primarily at the genus level. Fungal taxa that contained at
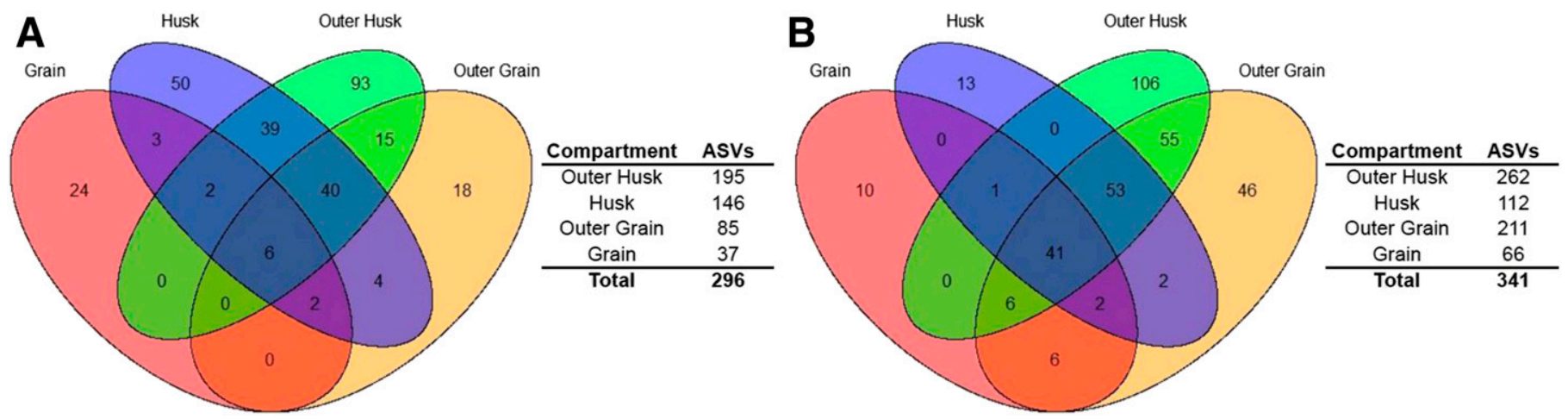

Fig. 2. Unique amplicon sequence variants (ASVs) Venn diagrams by seed compartment. Distribution of unique $\mathbf{A}$, bacterial and $\mathbf{B}$, fungal ASVs separated by seed compartment. Unique ASVs found in each seed compartment are shown next to the Venn diagram.
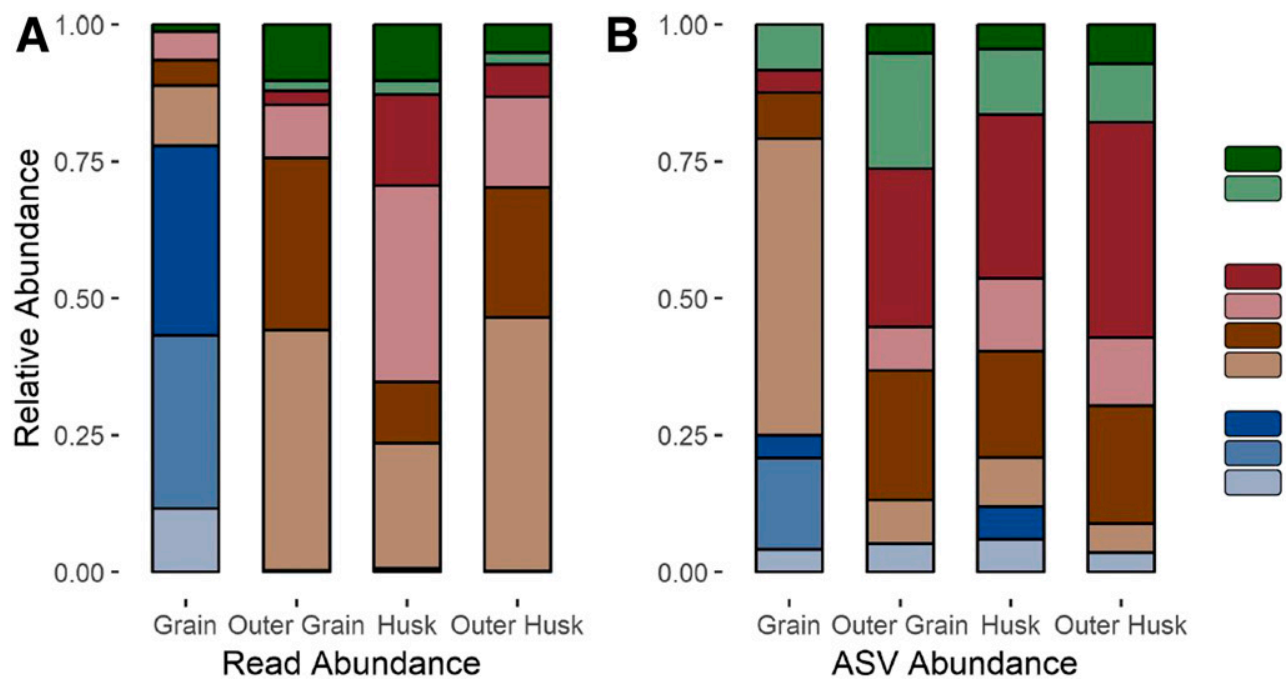

Taxa

L Actinobacteria

L Curtobacterium

L Microbacterium

L Proteobacteria

L Alphaproteobacteria

$\mathrm{L}$ Aureimonas

L Methylobacterium

L Rhizobium

$L$ Sphingomonas

L Gammaproteobacteria

L Enterobacteriaceae (Ambiguous)

L Atlantibacter

L Franconibacter

Fig. 3. Top bacterial taxa with $>1 \%$ total reads. Relative abundance plots of the bacterial taxa with greater than $1.0 \%$ of total read abundance after being pooled based on seed compartment. A, Relative read abundance belonging to the respective taxonomic classification. B, Abundance of unique amplicon sequence variants (ASVs) belonging to the taxonomic classifications. ASV counts (grain 25; outer grain 40; husk 66; and outer husk 58). 
least $1 \%$ of the total fungal reads were extracted of which thirteen were identified as containing $96.4 \%$ of the total reads (Fig. 5). The seed compartments were largely comprised of members of the phylum Ascomycota, primarily a single taxa class, the Dothidiomycetes, ranging from 46.3 to $97.5 \%$ in abundance. Largely absent from the grain were members of the phylum Basidiomycota; however, these were much more abundant in the seed surface compartments (outer grain and outer husk) where they represented large portions of the total read abundance (36.4 to $46.0 \%$ ). Additionally, although read abundance of Basidiomycota diminished considerably in the grain, members of the phylum maintained similar diversity as ASV relative abundance throughout the seed compartments.
The fungi representing at least $0.1 \%$ of the total reads within respective taxa were extracted (Supplementary Table S3) of which there were 20 members, then clusters were constructed (Fig. 4B) as above. Cluster B was among the most extreme, primarily confined to the outer grain, however it contained the single genera Naganishia with little information as to why it may be present in this space. Cluster A contained the largest number of fungal taxa at eight members divided between Basidiomycota and Ascomycota whose abundance was highest in the outer husk compartment. ASVs assigned to clusters $\mathrm{C}, \mathrm{E}$, and $\mathrm{F}$ were among those with $>1 \%$ of the total reads, and subsequently followed abundance trends that were apparent in Figure 5. ASVs in cluster D experienced an increased abundance

\section{A}

B

1.2

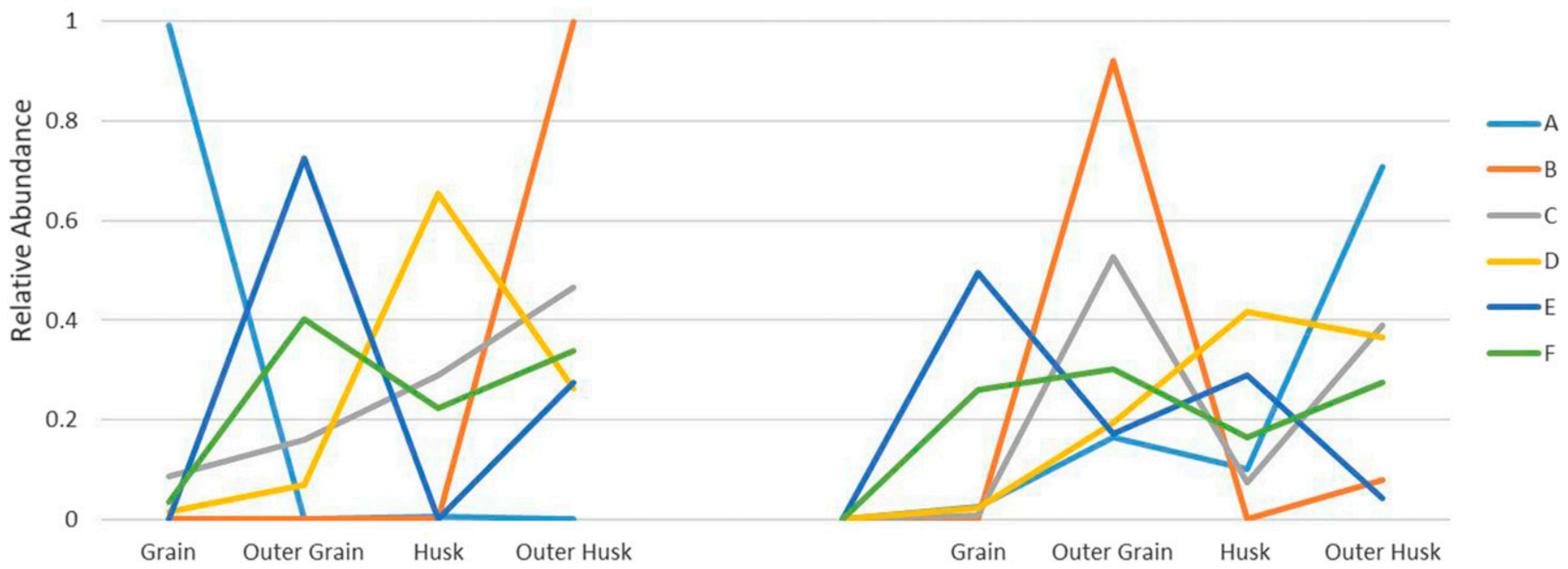

Fig. 4. Top bacterial and fungal taxa clusters with $>0.1 \%$ total read abundance. Clustering of the normalized relative abundance values for all $\mathbf{A}$, bacterial and $\mathbf{B}$, fungal taxa with greater than $0.1 \%$ of the total reads. Node values represent the average of the normalized abundance values within a cluster for each of the seed compartments.
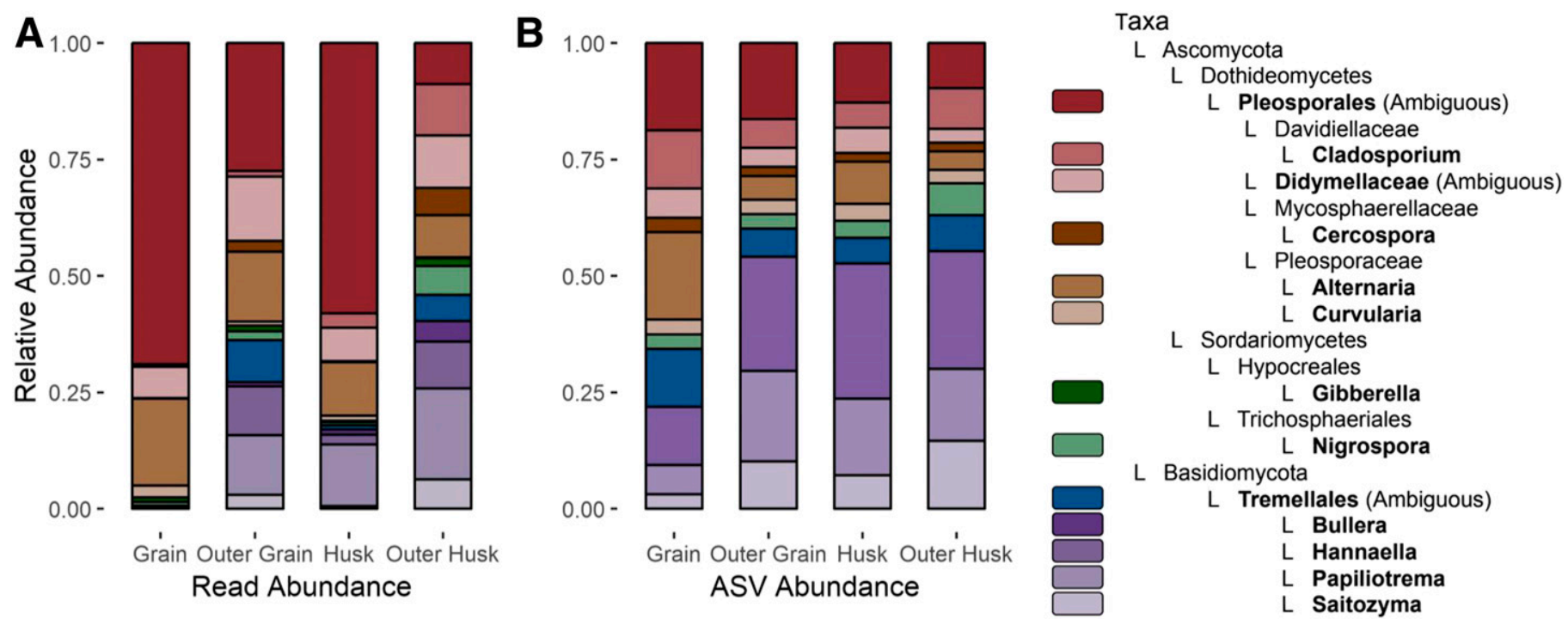

Fig. 5. Top fungal taxa with $>1 \%$ total reads. Relative abundance plots of the fungal taxa with greater than $1.0 \%$ of total read abundance after being pooled based on seed compartment. A, Relative read abundance belonging to the respective taxonomic classification. B, Abundance of unique amplicon sequence variants (ASVs) belonging to the taxonomic classifications. ASV counts (grain 32; outer grain 98; husk 55; and outer husk 103). 
from the inner- to the outermost compartments of the seed and contained equal members of the phylum Basidiomycota and order Pleosporales. Analysis by other experimental factors revealed additional features of interest. For example, Pleosporales were found throughout the seed, yet had a 30.4\% difference in abundance between seed sourced from 2013 and 2014. Additional disproportions were the $13.6 \%$ difference in abundance between locations for Alternaria, and $16.0 \%$ difference in abundance between genotypes for Papilotrema. Other differences in taxa representation were noted, especially when considering the seed year, although contributions to this effect remain unknown.

Rice seed diversity. To evaluate if the microbiomes formed distinct communities when grouped based on the four factors (seed compartment, year, location, and year), a PCA was first generated for the 24 samples for both the bacterial and fungal members (Fig. $6)$. The resulting analyses revealed that the samples, when colorized by seed compartment, were relatively distinct although this was much more apparent for the bacterial rather than fungal communities. For the bacterial PCA, the grain compartment formed the most distinct grouping. However, when the outer husk was removed from the analysis, the husk and outer grain compartments also formed relatively distinct groups from the others. For the fungal PCA, the grain, outer grain, and outer husk tissues formed distinct groupings with the husk overlapping all three. When the results from the PCA were colorized based on genotype, location, or seed year, no obvious separation was observed.

An additional approach was used to evaluate the seed microbiome diversity across seed compartment, year, genotype, and location utilizing packages in R. Shannon diversity, a measure of species diversity and evenness (Lemos et al. 2011), revealed a reduction in the diversity index from the outer to the innermost seed compartments for the fungi only (Table 2). For fungal taxa, greatest evenness was observed among taxa in the outer husk, and least in the grain, where members within the order Pleosporales dominated. In addition, there were sizeable differences in fungal diversity between seed samples separated by location and genotype with DB and M202 being more diverse, respectively. The bacteria exhibited the greatest diversity within the husk compartment with abundant members of the phylum Actinobacteria and class Alphaproteobacteria. These findings are consistent with the observation that microbial abundance and diversity tends to decrease from the epiphyte to the endophyte of most plant tissues, especially within the root (Edwards et al. 2015; Sánchez-Cañizares et al. 2017). In relation to the seed, this might suggest the presence of some type of selective mechanism that limits occupancy by certain microbes, such as the potential inability of certain microbes to penetrate tissues as postulated above.

Defining and characterizing a core rice seed microbiome. A key motivator for this research was to elucidate a core microbiome for rice seed, a set of microbes that one could expect to find within the seed independent of its genotype, harvest year, and location. To define the per-compartment seed core microbiome, the ASVs that were found in each seed sample for a compartment were identified. In the deduction of a core microbiome, it is often required that at least $90 \%$ of samples contain a particular taxa in order for it to be part of the core (Huse et al. 2012); however, due to the limited number of samples and potential for expansion with further data, we required each taxa be present in all samples of a particular compartment regardless of abundance. The ASVs identified in the bacterial core represented $13.4 \%$ of the bacterial ASVs, while the fungal core represented $12.2 \%$ of the fungal ASVs. We found both the bacterial core and the fungal core contained primarily members that were high in abundance with at least $0.1 \%$ of the total reads per seed compartment. The only exceptions were in the fungal core microbiome, where the genera Setophoma, Fusarium, Dioszegia, Papilotrema and Russulales were identified with $<0.1 \%$ read abundance. Core microbes found in a seed compartment occurring with less than $0.1 \%$ of the respective compartment abundance could be considered contaminants, or rare and conserved members of the rice microbiome.

Overall, the bacterial core contained 21 unique ASVs with most present in the husk (Table 3). The grain contained taxa that were not shared with any other compartment, all of which belonged to the family Enterobacteriaceae. Several Actinobacteria including Curtobacterium, and Microbacterium as well as Alphaproteobacteria such as Methylobacterium, Sphingomonas, Aureimonas, and Rhizobium were common to all seed compartments except the grain.

The fungal core rice microbiome consisted of a total of 39 unique ASVs identified to the genera level (Table 4). Core Ascomycota, particularly Dothidiomycetes were commonly associated with all 4 seed compartments, whereas Basidiomycota were notably absent from the grain. Among Ascomycota, the species Alternaria, Cercospora, and Cladosporium were prominent in all compartments, whereas Epicoccum and a few other taxa were found in the outer husk tissue exclusively. Several Basidiomycota including Hannaella, Papiltrema, and Tremellales were commonly observed in all compartments except the grain.
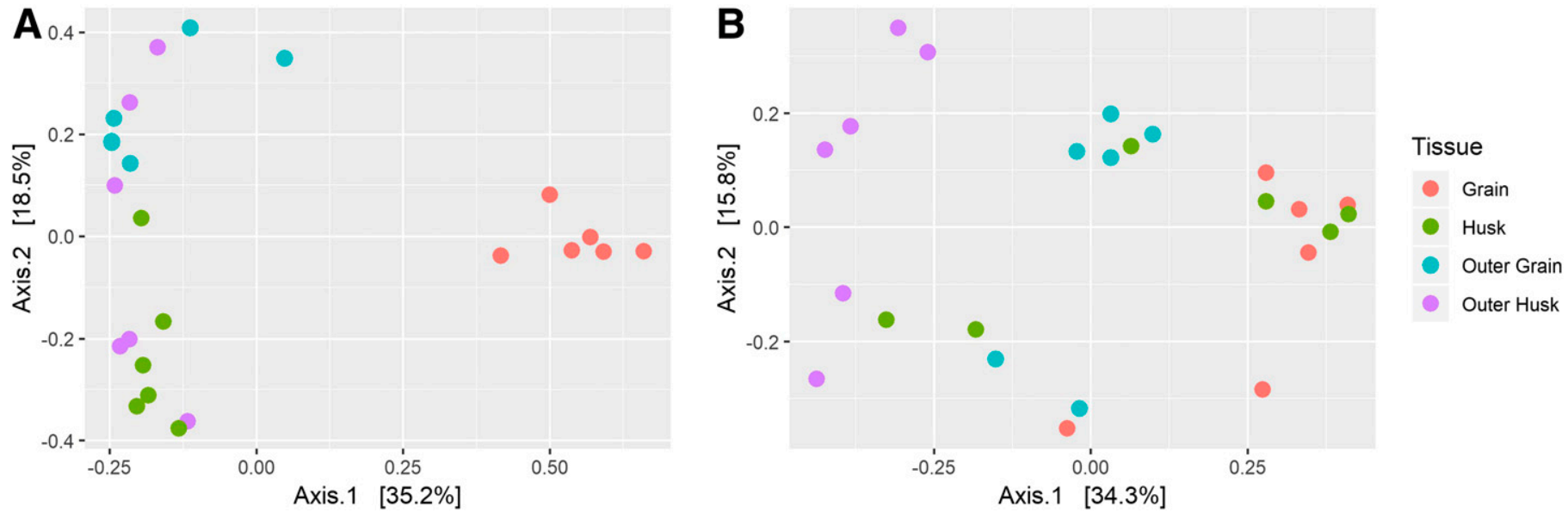

Fig. 6. Bacterial and fungal principal component analysis performed on the $24 \mathbf{A}$, bacterial and $\mathbf{B}$, fungal samples, and then colorized by seed compartment. 


\section{DISCUSSION}

It is currently recognized that there are many internal and external factors that shape the microbiome within plants. External factors such as weather, soil composition, nutrient supply, water, and others have a major effect, although the extent to which each have on the maturing plant microbiome remains unclear (Buyer et al. 1999; Hacquard 2016). To elucidate whether rice seeds possess a relatively stable core microbiome likely through vertical descent, we selected six different but related rice seeds from two different locations, genotypes, and years for this study. Extraction and analysis of each of the seeds broken down by four compartments yielded 296 unique bacterial and 341 unique fungal ASVs. Our diversity results showed that the seed compartment was the most impactful factor that distinguished samples for the bacterial communities. In general, we observed a decrease in unique ASVs from the exterior to the interior the seed, a trend commonly observed in plant tissues (Sánchez-Cañizares et al. 2017). Remarkably, the grain compartment exhibited more bacterial diversity as estimated by the Shannon diversity index than both the outer husk and outer grain despite the reduced number of taxa, suggesting that those few taxa that do occupy the inner compartment are at relatively even abundances.

TABLE 2

Shannon diversity of taxa within the samples pooled based on the four seed factors ${ }^{a}$

\begin{tabular}{lcc}
\hline Factor & Bacteria & Fungi \\
\hline Outer husk & 2.624 & 3.283 \\
\hline Husk & 3.088 & 2.028 \\
\hline Outer grain & 2.089 & 2.855 \\
\hline Grain & 2.822 & 1.208 \\
\hline Katy & 2.813 & 2.262 \\
\hline M202 & 3.048 & 2.743 \\
\hline DB & 2.740 & 2.720 \\
\hline UA & 3.252 & 2.137 \\
\hline 2013 & 2.790 & 2.685 \\
\hline 2014 & 3.032 & 2.307 \\
\hline
\end{tabular}

a $A$ higher value can indicate a greater number of species and/or more even relative abundance between the species.
For the fungal communities, a combination of seed compartment and year were critical factors. This finding may be a consequence of the unavailability of seed from the UA location for the year 2013; however, it was not reflected among the bacterial samples. If the lack of samples did not play an important role, this result may reflect effects of environmental changes or seed storage duration on the fungal communities within the rice seed (Lane et al. 2018). It is also noteworthy that nearly $2 / 3$ of fungi found in the grain compartment were shared with other compartments. This is in contrast to less than $1 / 6$ of the bacteria found in the grain being shared. This may suggest stronger selectivity for bacteria that occupy the grain of the seed or fungi have a greater ability to move through the entire seed. Regardless, for both bacteria and fungi the seed compartment had a consistently strong impact on the communities, suggesting the niches within the plant itself are the strongest shaping factor.

Specific microbes likely play important roles for nutrient supply and pathogen defense to promote plant performance. To assess perspectives on the individual members that comprise the microbiomes, we examined the microbes with more than $1 \%$ of the total reads and placed those with more than $0.1 \%$ of the total reads into clusters based on abundance trends. Our results revealed a number of microbes and associations that have been previously described in literature. For example, within the bacterial kingdom, the grain compartment was mainly composed of the family Enterobacteriaceae that often occupy the innermost plant tissues (Hardoim et al. 2013). On the other hand, as has been noted in other studies, a greater abundance of Alphaproteobacteria were detected within the outer compartments (Cottyn et al. 2009; Truyens et al. 2015). Asaia was present only the outer husk and may represent a contaminant due to its low abundance, although some isolates of the bacterium have been observed associated with fermenting glutinous rice (Yamada et al. 2000). Within the fungal kingdom, the grain was almost entirely dominated by Dothideomycetes, while the outer compartments contained an increasing abundance of Basidiomycota (Shade et al. 2017). Multiple ASVs could only be assigned to relatively high taxonomic ranks, such as family or order, which suggests there are multiple genera, species or subspecies within these compartments. This observation may be due to members of the latter mainly being isolated in free floating unicellular yeast forms in nature (De García et al. 2010; Nakase and Suzuki 1985; Nutaratat et al. 2014), which are unable to easily penetrate the grain. The usage of additional loci to identify the microbiome members may shed light on just how much diversity exists within each genera classification.

\begin{tabular}{|c|c|c|c|c|}
\hline \multicolumn{5}{|c|}{$\begin{array}{c}\text { TABLE } 3 \\
\text { Bacterial core seed microbiome }^{a}\end{array}$} \\
\hline Bacterial class & Grain & Outer grain & Husk & Outer husk \\
\hline \multirow{2}{*}{ Actinobacteria } & & & Curtobacterium $(-, 1, \mathbf{1},-)$ & \\
\hline & & & Kineococcus $(-,-, \mathbf{1},-)$ & \\
\hline \multirow{2}{*}{ Alphaproteobacteria } & & Rhizobium $(-, 1,1,1)$ & Methylobacterium $(-,-, \mathbf{1}, 1)$ & Aureimonas $(-, 1,1,1)$ \\
\hline & & Sphingomonas $(-, \mathbf{1}, 1,1)$ & Rhizobium $(-, 1,1,1)$ & Rhizobium $(-, 1,1, \mathbf{1})$ \\
\hline Proteobacteria & Enterobacteriaceae (3,-,-,-) & & & \\
\hline Gammaproteobacteria & Franconibacter $(1,-,-,-)$ & & & \\
\hline
\end{tabular}


The abundance trends for some of these microbes have interesting implications for inheritance mechanisms. For example, Microbacterium, Curtobacterium, Hannaella, and others were primarily found in the interstitial compartments (outer grain and outer husk) containing seed surfaces, suggesting these might be transferred to the seed through the xylem or other plant interspaces. Other organisms with the ability to transverse through plant tissues, such as bacteria and filamentous fungi through the action of cell wall degrading enzymes (Aparna et al. 2009; Kubicek et al. 2014), might be able to penetrate the seed tissue to occupy the grain or husk compartments. Further interrogation of inheritance mechanisms, such as detailed cytological analyses using microbes tagged with reporter genes, however, is beyond the scope of this study (Lübeck et al. 2002). The core microbiome in this study provides several ideal candidates for such research.

The primary focus of this research was to identify the core microbiome members for rice seed, a group of microbes that appear to be ubiquitously present in seed regardless of the impact of seed genotype, year, and location. These microbes are of interest for the development of agricultural products, as they more likely represent a conserved grouping that might thrive for multiple rice genotypes and over a large geographic area (Barret et al. 2015; Parnell et al. 2016). Our core contained 21 and 39 ASVs for the bacterial and fungal microbiomes, respectively. Review of literature for the bacterial core showed that $96.2 \%$ of the taxa represented in the core have, at least, been isolated and reported upon from rice seed (Bertani et al. 2016; Kumari et al. 2013; Midha et al. 2016). Additionally, many species of these reported genera have been cultured from various rice plants around the globe enabling their future investigation or modification. Taxa found in the grain compartment are of interest as they are typically confined to the endophyte of rice plants, and some species in the genera Enterobacter have been noted to have rice growth promoting effects through the supply of nitrogen and phosphorous (Hardoim et al. 2013). At high abundance among remaining seed compartments were a variety of Methylobacterium, Sphingomonas, and Aureimonas, which have been commonly found associated with rice plants and seed (Midha et al. 2016). Certain species of Sphingomonas have documented nitrogen fixing mechanisms and have been isolated from both rice seed and roots (Videira et al. 2009; Xie and Yokota 2006). Methylobacterium is a genera of interest to rice and the environment, as it is able to both fix nitrogen and oxidize methane. It is notable that wetland rice is responsible for releasing $\sim 20 \%$ of the world's methane content annually (Tani et al. 2015). Rhizobium is commonly associated with plant growth promotion of legumes through the fixation of nitrogen, however its association and function with rice remains ambiguous. Finally, species found in Curtobacterium, Aureimonas, and Microbacterium have been isolated from rice although there have been no speculated or studied function (Hardoim et al. 2012; Kumari et al. 2013; Madhaiyan et al. 2013).

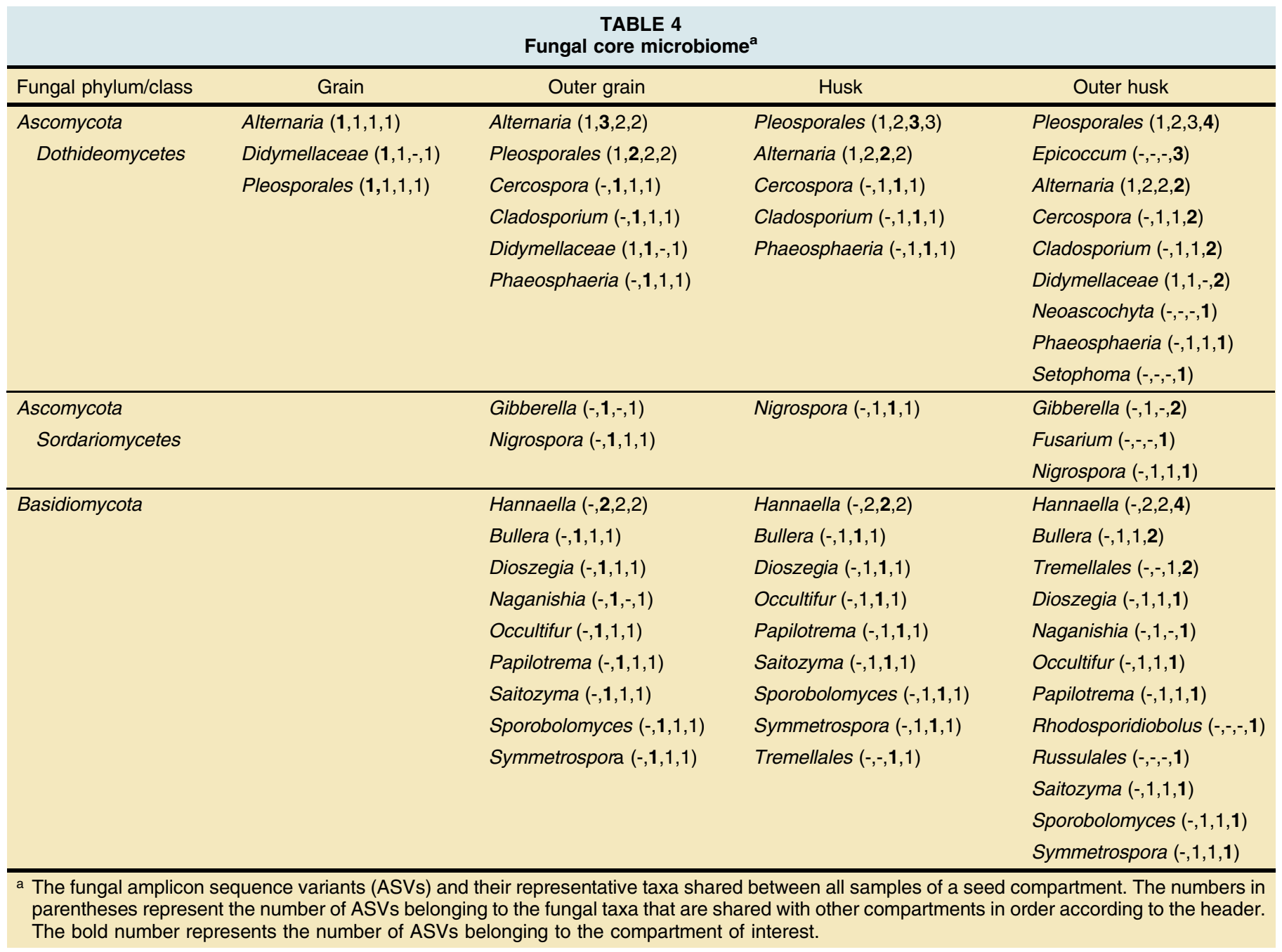


Within the fungal core microbiome, Alternaria was among the most prominent genera that has been commonly reported in rice, noted to produce possibly active metabolites (Wang et al. 2014). Additionally some species within the genera are rice pathogens, such as $A$. padwickii, a common leaf spot pathogen of rice (Gutierrez et al. 2010). Cladosporium was also identified in abundance among the outer compartments of the seed, species of which have been noted to have suppressing effects on rice blast disease when applied before or during infection by Magnaporthe oryzae, which causes major reductions of rice yield annually (Chaibub et al. 2016). Additionally, within the phylum Ascomycota, species within the genera Gibberella have been observed in a species complex with Fusarium, suggesting their mutual abundance in the core (Hsuan et al. 2011). Finally, within the phylum Basidiomycota few documented functions for the various fungi identified have been noted, however most that have been isolated have been reported in unicellular yeast forms which as noted above may account for their presence within the porous outer seed compartments (Landell et al. 2014; Nakase and Suzuki 1985; Robert et al. 2018).

Additional cultivars would provide the depth of investigation needed to validate and expand the value of our findings for future research and product development. The primary theoretical issue with this pursuit is that as more plant genotypes or conditions are included in the study, the size of the core may approach zero. There are some workarounds such as relaxing the taxonomic classifications allowing for more individuals to be propagated into the core at a cost of resolution, or simply to construct the core and redefine it depending on the genotypic or geographic context of interest. While there were numerous accounts of bacteria, the study of fungi as members of the plant microbiome is still in its infancy for many plant species and few examples are available. More importantly, the limited resolution for the unidentified taxonomic classifications of fungi makes this challenging (Tedersoo and Lindahl 2016). While the core microbiome presented in this study represents only a small subset of the world rice population, our work provides a foundation into what such a community looks like for rice seed.

\section{ACKNOWLEDGMENTS}

We thank members of the Center for Integrated Fungal Research for their interest in this work and Y. Jia, USDA Dale Bumpers National Rice Research Center, for providing the seeds used this study.

\section{LITERATURE CITED}

Abarenkov, K., Nilsson, R. H., Larsson, K. H., Alexander, I. J., Eberhardt, U., Erland, S., et al. 2010. The UNITE database for molecular identification of fungi-Recent updates and future perspectives. New Phytol. 186:281-285.

Aparna, G., Chatterjee, A., Sonti, R. V., and Sankaranarayanan, R. 2009. A cell wall-degrading esterase of Xanthomonas oryzae requires a unique substrate recognition module for pathogenesis on rice. Plant Cell Online 21:1860-1873.

Bacilio-Jiménez, M., Aguilar-Flores, S., Del Valle, M. V., Pérez, A., Zepeda, A., and Zenteno, E. 2001. Endophytic bacteria in rice seeds inhibit early colonization of roots by Azospirillum brasilense. Soil Biol. Biochem. 33:167-172.

Baldwin, K., Dohlman, E., Childs, N., and Foreman, L. 2011. Consolidation and structural change in the US rice sector. U.S. Dep. Agric. 1-28. https:// www.ers.usda.gov/publications/pub-details/?pubid=38492

Barret, M., Briand, M., Bonneau, S., Preveaux, A., Valiere, S., Bouchez, O., et al. 2015. Emergence shapes the structure of the seed microbiota. Appl. Environ. Microbiol. 81:1257-1266.

Beckstead, J., Meyer, S. E., Molder, C. J., and Smith, C. 2007. A race for survival: Can Bromus tectorum seeds escape Pyrenophora semeniperdacaused mortality by germinating quickly? Ann. Bot. 99:907-914.
Bertani, I., Abbruscato, P., Piffanelli, P., Subramoni, S., and Venturi, V. 2016. Rice bacterial endophytes: Isolation of a collection, identification of beneficial strains and microbiome analysis. Environ. Microbiol. Rep. 8: 388-398.

Breidenbach, B., Pump, J., and Dumont, M. G. 2016. Microbial community structure in the rhizosphere of rice plants. Front. Microbiol. 6:1-12.

Bulgarelli, D., Rott, M., Schlaeppi, K., Ver Loren van Themaat, E., Ahmadinejad, N., Assenza, F., et al. 2012. Revealing structure and assembly cues for Arabidopsis root-inhabiting bacterial microbiota. Nature 488:91-95.

Bulgarelli, D., Schlaeppi, K., Spaepen, S., van Themaat, E. V. L., and SchulzeLefert, P. 2013. Structure and functions of the bacterial microbiota of plants. Annu. Rev. Plant Biol. 64:807-838.

Buyer, J. S., Roberts, D. P., and Russek-Cohen, E. 1999. Microbial community structure and function in the spermosphere as affected by soil and seed type. Can. J. Microbiol. 45:138-144.

Callahan, B. J., McMurdie, P. J., Rosen, M. J., Han, A. W., Johnson, A. J. A., and Holmes, S. P. 2016. DADA2: High-resolution sample inference from Illumina amplicon data. Nat. Methods 13:581-583.

Chaibub, A. A., de Carvalho, J. C. B., de Sousa Silva, C., Collevatti, R. G., Gonçalves, F. J., de Carvalho Barros Côrtes, M. V., et al. 2016. Defense responses in rice plants in prior and simultaneous applications of Cladosporium sp. during leaf blast suppression. Environ. Sci. Pollut. Res. 23: 21554-21564.

Chen, H., and Boutros, P. C. 2011. VennDiagram: A package for the generation of highly-customizable Venn and Euler diagrams in R. BMC Bioinformatics 12.

Compant, S., Clément, C., and Sessitsch, A. 2010. Plant growth-promoting bacteria in the rhizo- and endosphere of plants: Their role, colonization, mechanisms involved and prospects for utilization. Soil Biol. Biochem. 42: 669-678.

Cottyn, B., Debode, J., Regalado, E., Mew, T. W., and Swings, J. 2009. Phenotypic and genetic diversity of rice seed-associated bacteria and their role in pathogenicity and biological control. J. Appl. Microbiol. 107:885-897.

De García, V., Brizzio, S., Russo, G., Rosa, C. A., Boekhout, T., Theelen, B., et al. 2010. Cryptococcus spencermartinsiae sp. nov., a basidiomycetous yeast isolated from glacial waters and apple fruits. Int. J. Syst. Evol. Microbiol. 60:707-711.

Edwards, J., Johnson, C., Santos-Medellín, C., Lurie, E., Podishetty, N. K., Bhatnagar, S., et al. 2015. Structure, variation, and assembly of the rootassociated microbiomes of rice. Proc. Natl. Acad. Sci. 112:E911-E920.

Engelbrektson, A., Kunin, V., Engelbrektson, A., Kunin, V., Glavina del Rio, T., Hugenholtz, P., et al. 2012. Defining the core Arabidopsis thaliana root microbiome. Nature 488:86-90.

Fadrosh, D. W., Ma, B., Gajer, P., Sengamalay, N., Ott, S., Brotman, R. M., et al. 2014. An improved dual-indexing approach for multiplexed 16S rRNA gene sequencing on the Illumina MiSeq platform. Microbiome 2:6.

Fitzpatrick, C. R., Copeland, J., Wang, P. W., Guttman, D. S., Kotanen, P. M., and Johnson, M. T. J. 2018. Assembly and ecological function of the root microbiome across angiosperm plant species. Proc. Natl. Acad. Sci. 115: E1157-E1165.

Fukuyama, J. 2017. Adaptive gPCA: A method for structured dimensionality reduction. arXiv:1702.00501.

Goggin, D. E., Emery, R. J. N., Kurepin, L. V., and Powles, S. B. 2015. A potential role for endogenous microflora in dormancy release, cytokinin metabolism and the response to fluridone in Lolium rigidum seeds. Ann. Bot. 115:293-301.

Gundel, P. E., Rudgers, J. A., and Ghersa, C. M. 2011. Incorporating the process of vertical transmission into understanding of host-symbiont dynamics. Oikos 120:1121-1128.

Gutierrez, S. A., Carmona, M. A., and Reis, E. M. 2010. Methods for detection of Alternaria padwickii in rice seeds. J. Phytopathol. 158:523-526.

Hacquard, S. 2016. Disentangling the factors shaping microbiota composition across the plant holobiont. New Phytol. 209:454-457.

Hamid, M. I., Hussain, M., Wu, Y., Zhang, X., Xiang, M., and Liu, X. 2017. Successive soybean-monoculture cropping assembles rhizosphere microbial communities for the soil suppression of soybean cyst nematode. FEMS Microbiol. Ecol. 93:1-10.

Hardoim, P. R., Hardoim, C. C. P., van Overbeek, L. S., and van Elsas, J. D. 2012. Dynamics of seed-borne rice endophytes on early plant growth stages. PLoS One 7:e30438.

Hardoim, P. R., Nazir, R., Sessitsch, A., Elhottová, D., Korenblum, E., Van Overbeek, L. S., et al. 2013. The new species Enterobacter oryziphilus sp. nov. and Enterobacter oryzendophyticus sp. nov. are key inhabitants of the endosphere of rice. BMC Microbiol. 13:164. 
Hsuan, H. M., Salleh, B., and Zakaria, L. 2011. Molecular identification of Fusarium species in Gibberella fujikuroi species complex from rice, sugarcane and maize from Peninsular Malaysia. Int. J. Mol. Sci. 12: 6722-6732.

Huang, Y., Kuang, Z., Wang, W., and Cao, L. 2016. Exploring potential bacterial and fungal biocontrol agents transmitted from seeds to sprouts of wheat. Biol. Control 98:27-33.

Huse, S. M., Ye, Y., Zhou, Y., and Fodor, A. A. 2012. A core human microbiome as viewed through $16 \mathrm{~S}$ rRNA sequence clusters. PLoS One 7: e34242.

Kawahara, Y., de la Bastide, M., Hamilton, J. P., Kanamori, H., McCombie, W. R., Ouyang, S., et al. 2013. Improvement of the Oryza sativa Nipponbare reference genome using next generation sequence and optical map data. Rice (N. Y.) 6:4-10.

Kubicek, C. P., Starr, T. L., and Glass, N. L. 2014. Plant cell wall-degrading enzymes and their secretion in plant-pathogenic fungi. Annu. Rev. Phytopathol. 52:427-451.

Kumari, P., Bandyopadhyay, S., and Das, S. K. 2013. Microbacterium oryzae sp. nov., an actinobacterium isolated from rice field soil. Int. J. Syst. Evol. Microbiol. 63:2442-2449.

Landell, M. F., Brandão, L. R., Barbosa, A. C., Ramos, J. P., Safar, S. V. B., Gomes, F. C. O., et al. 2014. Hannaella pagnoccae sp. nov., a tremellaceous yeast species isolated from plants and soil. Int. J. Syst. Evol. Microbiol. 64: 1970-1977.

Lane, B., Sharma, S., Niu, C., Maina, A. W., Wagacha, J. M., Bluhm, B. H., et al. 2018. Changes in the fungal microbiome of maize during hermetic storage in the United States and Kenya. Front. Microbiol. 9:1-13.

Lemos, L. N., Fulthorpe, R. R., Triplett, E. W., and Roesch, L. F. W. 2011. Rethinking microbial diversity analysis in the high throughput sequencing era. J. Microbiol. Methods 86:42-51.

Lübeck, M., Knudsen, I. M. B., Jensen, B., Thrane, U., Janvier, C., and Funck Jensen, D. 2002. GUS and GFP transformation of the biocontrol strain Clonostachys rosea IK726 and the use of these marker genes in ecological studies. Mycol. Res. 106:815-826.

Madhaiyan, M., Hu, C. J., Jegan Roy, J., Kim, S. J., Weon, H. Y., Kwon, S. W., et al. 2013. Aureimonas jatrophae sp. nov. and Aureimonas phyllosphaerae sp. nov., leaf-associated bacteria isolated from Jatropha curcas. L. Int. J. Syst. Evol. Microbiol. 63:1702-1708.

Midha, S., Bansal, K., Sharma, S., Kumar, N., Patil, P. P., Chaudhry, V., et al. 2016. Genomic resource of rice seed associated bacteria. Front. Microbiol. 6: $1-8$.

Nakase, T., and Suzuki, M. 1985. Ballistospore-forming yeasts found on the surface of the Japanese rice plant, Oryza sativa L. J. Gen. Appl. Microbiol. 31: 457-474.

Nelson, E. B. 2004. Microbial dynamics and interactions in the spermosphere. Annu. Rev. Phytopathol. 42:271-309.

Nutaratat, P., Srisuk, N., Arunrattiyakorn, P., and Limtong, S. 2014. Plant growth-promoting traits of epiphytic and endophytic yeasts isolated from rice and sugar cane leaves in Thailand. Fungal Biol. 118:683-694.

Oksanen, J. 2015. Multivariate analysis of ecological communities in R: Vegan tutorial. R Doc. 1-43.
Okunishi, S., Sako, K., Mano, H., Imamura, A., and Morisaki, H. 2005. Bacterial flora of endophytes in the maturing seed of cultivated rice (Oryza sativa). Microbes Environ. 20:168-177.

Parnell, J. J., Berka, R., Young, H. A., Sturino, J. M., Kang, Y., Barnhart, D. M., et al. 2016. From the lab to the farm: An industrial perspective of plant beneficial microorganisms. Front. Plant Sci. 7:1-12.

Quast, C., Pruesse, E., Yilmaz, P., Gerken, J., Schweer, T., Yarza, P., et al. 2013. The SILVA ribosomal RNA gene database project: Improved data processing and web-based tools. Nucleic Acids Res. 41:D590-D596.

Robert, V., Stegehuis, G., and Stalpers, J. 2018. MycoBank database. http:// www.mycobank.org/

Sánchez-Cañizares, C., Jorrín, B., Poole, P. S., and Tkacz, A. 2017. Understanding the holobiont: The interdependence of plants and their microbiome. Curr. Opin. Microbiol. 38:188-196.

Shade, A., Jacques, M. A., and Barret, M. 2017. Ecological patterns of seed microbiome diversity, transmission, and assembly. Curr. Opin. Microbiol. 37:15-22.

Tani, A., Sahin, N., Fujitani, Y., Kato, A., Sato, K., and Kimbara, K. 2015. Methylobacterium species promoting rice and barley growth and interaction specificity revealed with whole-cell matrix-assisted laser desorption/ ionization-time of-flight mass spectrometry (MALDI-TOF/MS) analysis. PLoS One 10:e0129509.

Tedersoo, L., and Lindahl, B. 2016. Fungal identification biases in microbiome projects. Environ. Microbiol. Rep. 8:774-779.

Truyens, S., Weyens, N., Cuypers, A., and Vangronsveld, J. 2015. Bacterial seed endophytes: Genera, vertical transmission and interaction with plants. Environ. Microbiol. Rep. 7:40-50.

Usyk, M., Zolnik, C. P., Patel, H., and Levi, M. H. 2017. Novel ITS1 fungal primers for characterization of the mycobiome. MSphere 2:e00488-17.

Vandenkoornhuyse, P., Quaiser, A., Duhamel, M., Le Van, A., and Dufresne, A. 2015. The importance of the microbiome of the plant holobiont. New Phytol. 206:1196-1206.

Videira, S. S., De Araujo, J. L. S., Da Silva Rodrigues, L., Baldani, V. L. D., and Baldani, J. I. 2009. Occurrence and diversity of nitrogen-fixing Sphingomonas bacteria associated with rice plants grown in Brazil. FEMS Microbiol. Lett. 293:11-19.

Walters, W., Hyde, E. R., Berg-lyons, D., Ackermann, G., Humphrey, G., Parada, A., et al. 2015. Improved bacterial 16S rRNA gene (V4-V5) and fungal internal transcribed spacer marker gene primers for microbial community surveys. mSystems 1:1-11.

Wang, Y., Yang, M. H., Wang, X. B., Li, T. X., and Kong, L. Y. 2014. Bioactive metabolites from the endophytic fungus Alternaria alternata. Fitoterapia 99: 153-158.

Wickham, H., Chang, W., Henry, L., Lin Pedersen, T., Takahashi, K., Wilke, C., et al. 2018. Package 'ggplot2'-Create Elegant Data Visualisations Using the Grammar of Graphics. R Package.

Xie, C. H., and Yokota, A. 2006. Sphingomonas azotifigens sp. nov., a nitrogenfixing bacterium isolated from the roots of Oryza sativa. Int. J. Syst. Evol. Microbiol. 56:889-893.

Yamada, Y., Katsura, K., Kawasaki, H., Widyastuti, Y., Saono, S., Seki, T., et al. 2000. Asaia bogorensis gen. nov., sp. nov., an unusual acetic acid bacterium in the alpha-Proteobacteria. Int. J. Syst. Evol. Microbiol. 50:823-829. 